Musées, Patrimoine et Culture scientifiques et techniques

149 | 2013

septembre-octobre 2013

\title{
Les personnes âgées dépendantes : un nouveau public pour la CSTI?
}

Pierre-Marie Verjus et Philippe De Pachtère

\section{(2) OpenEdition \\ Journals}

Édition électronique

URL : http://journals.openedition.org/ocim/1271

DOI : 10.4000/ocim.1271

ISSN : 2108-646X

Éditeur

OCIM

Édition imprimée

Date de publication : 27 septembre 2013

Pagination : 11-15

ISSN : 0994-1908

Référence électronique

Pierre-Marie Verjus et Philippe De Pachtère, «Les personnes âgées dépendantes : un nouveau public pour la CSTI ? », La Lettre de I'OCIM [En ligne], 149 | 2013, mis en ligne le 27 septembre 2015, consulté le 19 avril 2019. URL : http://journals.openedition.org/ocim/1271 ; DOI : 10.4000/ocim.1271 


\title{
Les personnes âgées dépendantes: un nouveau public pour la CSTI?
}

\author{
Pierre-Marie Verjus et Philippe De Pachtère *
}

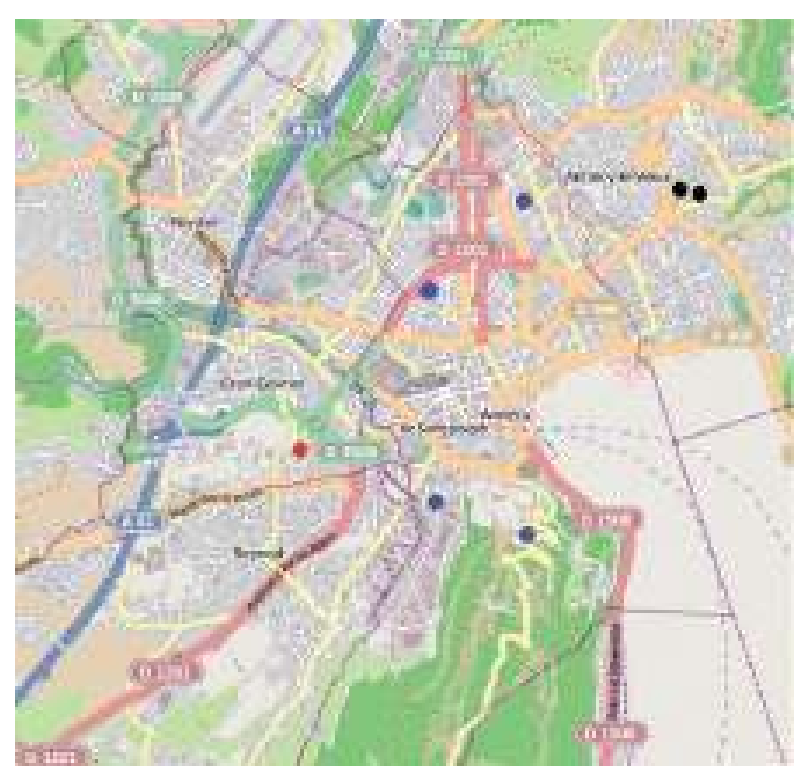

Carte d'implantation des 7 EHPAD et foyers pour personnes âgées gérés par le CIAS de la Communauté de l'agglomération d'Annecy (4 établissements à Annecy, 2 à Annecy-le-Vieux, 1 à Cran-Gevrier). o CCSTI La Turbine

* Pierre-Marie Verjus est médiateur scientifique au CCSTI la Turbine pmverjus@agglo-annecy.fr Philippe De Pachtère est directeur du CCSTI La Turbine pdepachtere@agglo-annecy.fr
Depuis 2006, La Turbine - le CCSTI de

Cran-Gevrier en Haute-Savoie - met en

œuvre un ensemble d'actions de médiation

scientifique adapté aux contraintes d'un

public âgé dépendant et résidant dans des

établissements spécialisés de l'agglomération :

les responsables dressent le bilan de l'opération

et proposent des perspectives d'évolution de

cette pratique.

\section{Contexte et enjeux}

Le Centre de Culture Scientifique, Technique et Industrielle (CCSTI) La Turbine est un des dix établissementsculturels delaCommunautédel'agglomération d'Annecy (C2A). Situé à Cran-Gevrier, il constitue un lieu d'exposition, d'échanges et de rencontres, et s'adresseà tous les publics, néophytes ou spécialistes, et de tout âge.

Comme tout établissement culturel public, une des missions du CCSTI visant à entrer dans le processus de démocratisation de la Culture cher à André Malraux, est de rendre accessible la culture scientifique, technique et industrielle au plus grand nombre.

Pour atteindre cet objectif et sensibiliser le public le plus large aux sciences, le CCSTI La Turbine propose, toutaulongdel'année, desactions demédiationscientifique en son sein, mais également d'autres interventions hors-les-murs.

Dans ce cadre, et depuis l'ouverture du CCSTI fin 2004, nous constatons que nos offres de culture scientifiqueproposéesintéressentunnombrenonnégligeable de personnes âgées indépendantes et valides. Notre volonté constante d'élargissement de nos publics, en 
terme de diversité, d'identification et de recherche de nouveaux publics, a conduit l'équipe du CCSTI dès 2006 - alors que l'établissement venait d'être transféré à la C2A - à s'inscrire dans un processus d'action/ réflexion sur la mise en œuvre d'actions de médiation scientifiqueà destination de personnesâgées dépendantes, accueillies dans différentsétablissements dans le cadre du CIAS de la C2A.

La pertinence de poursuivre et de développer de telles actions apparaît vite comme une évidence tant les enjeuxsemblaientimportantspourlepublicenvisagé: mise en valeur des connaissances de chacun - et donc de la personne -, création de lien social et intergénérationnel, travail de la mémoire, aide au maintien des facultés psychomotrices...

\section{Démarches et résultats}

Une première collaboration a été initiée à l'occasion de la Fête de la Science en 2006 entre le CCSTI et le Centre Intercommunal d'Actions Sociales (CIAS) de la Communauté de l'Agglomération d'Annecy (C2A), collectivité territoriale qui porte également structurellement le CCSTI. L'action de médiation priorisée alors en concertation avec les animatrices des EHPAD a été la projection d'un film à caractère scientifique par un médiateur du CCSTI, suivie d'un temps d'échange avec les résidents des sept EHPAD et foyers gérés par la C2A.

Lesfilms projetésétaient cetteannée-là des documentaires sur la faune marine pour construire les premiers liens avec nos expositions.

Ce choix d'intervention a été défini en concertation avec les animatrices, notamment en fonction des capacités d'attention des résidents, et a permis de caler aussi bien les durées que l'accessibilité aux contenus. La durée totale de chaque intervention selon les établissements et les publics se situe entre 60 et 90 minutes, avec 45 minutes maximum de projection.

Parallèlement, il a été décidé, d'un commun accord entre les chefs des services concernés, que les actions de médiation menées par le CCSTI dans les établissements du CIAS seraient gratuites, alors qu'elles sont d'ordinaire tarifées.

Cette première expérience s'est avérée très positive en tout point (128 résidents et 14 agents des EHPAD et foyerstouchés),ets'estaccompagnéed'unenthousiasme unanimementexpriméparlespersonnesimpliquées. L'opération a doncété reconduite lors deséditions suivantes de la Fête de la Science, avec un succès constant: une moyenne de 130 personnes touchées pour chaque cycle d'interventions.

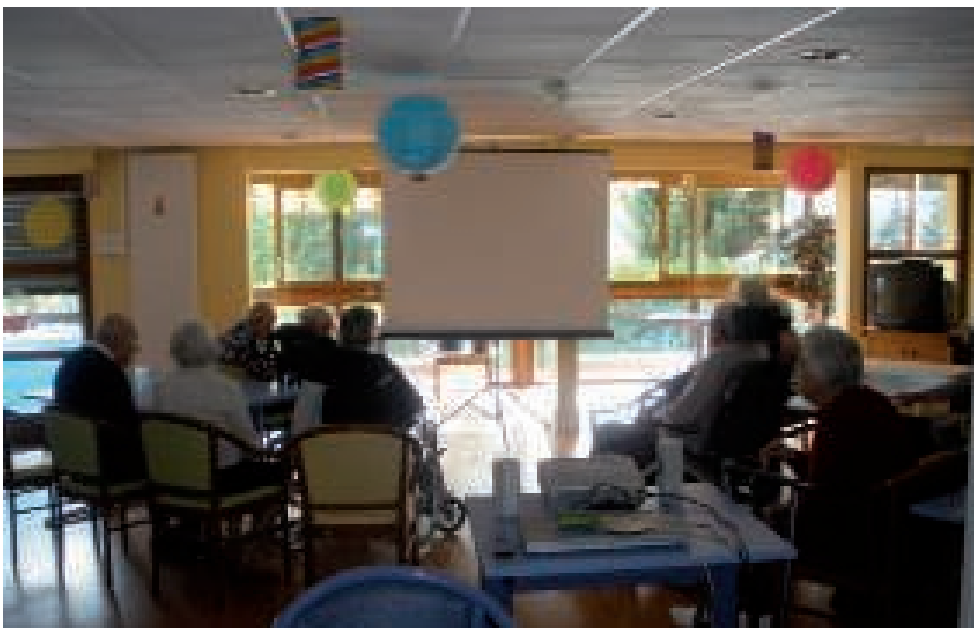

Intervention à la résidence Les Vergers (Annecy-le-Vieux) en 2012. Cette résidence a la particularité d'accueillir des résidents fortement dépendants, dont la plupart souffrent de la maladie d'Alzheimer. o CCSTI La Turbine

\section{Évolution}

En 2009, après plusieurs éditions de la Fête de la Science et dans le cadre d'une concertation permanente avec le personnel des EHPAD pour répondre au souhait de certaines animatrices, le CCSTI a élargi ses périodes d'interventions: des projections de films scientifiques ont ainsi été programmées, tout au long de l'année, dans tous les établissements pour personnes âgées.

Si le format de ces interventions est globalement resté identique, le contenu des films scientifiques projetés a lui, en revanche, évolué. Ainsi, dans la mesure des disponibilités du fonds documentaire du CCSTI, et toujours en étroite concertation avec les animatrices des résidences, il a été décidé de projeter des documentaires en lien avec les thématiques des expositions présentées à la Turbine : astronomie, urbanisme, environnement, apiculture..., un moyen d'intégrer ces actions hors les murs et d'impliquer les publics concernés dans le projet culturel et scientifique pluriannuel de notre établissement.

Au total, ce sont 664 personnes qui, entre 2006 et 2011 , ont bénéficié d'interventions mises en place par le CCSTI, pendant et hors Fête de la Science.

Commenousl'espérions depuisledébut, cesinterventions dans les établissements ont suscité la curiosité des résidents, au point que certains d'entre eux ont souhaité visiter les expositions présentées au CCSTI. Des visites spécifiques (temps de visites adaptés, mise à disposition de sièges, gratuité) sont alors organisées, 
et entre 2010 et 2012, ce sont sept groupes de personnes âgées (soit 61 personnes) dépendantes mais valides, qui se sont rendus au CCSTI afin de visiter, accompagnésd'unmédiateurscientifique, lesexpositions Sur les traces de l'eau, Le Roi Sommeil, Manivelles \& Roues dentées et L'eau y es-tu?

Rappelons à ce point que, au titre d'une collaboration inter services de la C2A, toutes les animations (dans et hors les murs) réalisées depuis 2006 par le CCSTI en faveur des résidents des EHPAD et foyers de l'agglomération, sont entièrement gratuites pour leurs bénéficiaires.

Pour le premier semestre 2013, deux groupes de résidentsontd'oresetdéjà réservédesvisitesd'expositions au CCSTI, et huit projections de films documentaires enétablissementsontétéprogramméessurlapériode d'avril à juin.

Là encore, la transposition de l'expérience acquise hors les murs, dans nos murs, a procuré enthousiasme et satisfaction,notammentgrâceauxthématiquesproposées et à la grande interactivité des expositions. Ce dernier point qui, sans avoir été étudié de manière systématique et encore moins statistique compte tenu de la taille de l'échantillon, a toutefois guidé nos réflexions sur l'évolution de nos pratiques dans les établissements.

\section{Perspectives}

En faisant un nouvel état des lieux, au niveau de la progressiondenoscompétencesetdenoscapacitésà proposer denouveauxoutils de médiation, mais aussi

\section{Témoignages recueillis auprès d'une animatrice et de trois résidents}

Valérie (animatrice)

«Beaucoup d'animations et d'ateliers divers sont programmés tout au long de l'année pour nos résidents: jeux de société, informatique, poésie, arts plastiques, conférences...

Les interventions proposées par le CCSTI s'intègrent parfaitementà la logique de notre programmation, et elles apportent un véritable «bonus» dans notre offre carellestouchentà différentsdomaines.Lefaitquenos résidentspuissentseretrouverpourregarderundocumentaire et en discuter ensuite avec le médiateur, et aussientreeux,représenteunmomentdedétenteetde distraction, mais aussi un temps qui demande de la concentration, parfoisfaisantappelàdessouvenirspersonnels ou collectifs. C'est aussi un moment de vie en société fort, car en plus du partage, on touche à des notions comme l'écoute et le respect de l'autre, mais aussi la confiance en soi, voire le dépassement des appréhensions par la prise de parole...

Le partenariat avec La Turbine fonctionne très bien depuissamiseen placeetnosrésidentssonttoujoursaussi satisfaitsqu'audébutdestempsd'interventionsproposés. Maisauvudescompétencesetdesoutilsdontdisposele CCSTI,je pense qu'il serait possible de diversifier les animations, avecdesateliersd'expérimentationsparexemple,ouenutilisantl'informatique.C'estlegenredechoses dontnosrésidentssontfriands, etquipermettraientd'explorer d'autres modes d'apport de la connaissance ».
Odette (résidente)

"J'aime bien parce que les films ne sont pas longs et qu'ilsmontrent des bellesimages. Deschosesqu'on connaît ou qu'on ne connaît pas, mais qui sont intéressantesetquinousapprennentbeaucoup.Etpuissurtout on peut poser des questions après. C'est important car d'habitudequandonregardedesdocumentairesaucoin salon,onn'apersonnequipeutrépondreànosquestions et nous aider quand on ne comprend pas. »

\section{Pierre (résident)}

«D'habitude,ondiscutetoujoursdesmêmeschoses, de ce qui se passe à la résidence, des nouvelles qu'on lit dans le journal... Là on peut discuter d'autres choses, même de choses dont on n'avait pas entendu parler avant de voir le film ! Ça permet aussi de comprendre deschosesqu'onentendàlatélécommelapollutionde l'eau et le changement declimat. On se rappelle dece que nous a dit le conférencier de la Turbine. On peut même en reparler avec nos petits-enfants ».

\section{Cécile (résidente)}

«Quel que soit le sujet, c'est toujours intéressant. On comprend ce qui est expliqué dans les films parce que c'est simple. Et puis on est vieux, on voit et on entend moins bien, alors le grand écran et les haut-parleurs, c'est vraiment bien pour nous. Ce qui me plaît bien aussi, c'est quelemonsieurvient de La Turbineetqu'on peut aussi aller là-bas voir les expositions. Quand il vientà la résidence, il nous donnetoujours desaffiches et nous parle des expositions. Ça donne envie d'y aller et ensuite Valérie (l'animatrice NDLR) nous y emmène. Ça fait une sortie. Et puis on y voit des choses intéressantes,qu'onseraitpeut-êtrejamaisallévoirautrement». 
concernant l'évolution de la situation des structures d'accueilpourpersonnesâgées, troisgrandesperspectives évidentes se sont imposées à nous :

- la première possibilité a été de toucher un plus grand nombrederésidentsen proposantnotreoffreexistante de médiation à d'autres établissements : en effet, depuis 2007, quatre nouveaux EHPAD ont été construits etontouvertleurs portesà Annecy, Argonay, Meythet et Poisy. L'ouverture des ces structures a ainsi porté à 769 (contre 459 auparavant) le nombre de personnes âgées résidanten établissements médicalisés sur leterritoire de l'agglomération ;

- la seconde perspective a été de poursuivre nos interventions dans les sept mêmes établissements spécialisés, mais en diversifiant et en enrichissant nos pratiques demédiation en direction des personnesâgées, dans le double but d'éviter toute lassitude éventuelle des résidents fidèles à nos animations, et d'essayer, par ce renouvellement d'offre, de toucher un nombre accru de participants ;

- la troisième solution envisageable résidait, quant à elle, en un savant mixage des deux premières.

C'est finalement la seconde piste que l'équipe du CCS$\mathrm{Tl}$ a décidé d'explorer :

- d'une part, parce que les ressources humaines, matérielles, techniques et temporelles dont dispose l'équipe de médiateurs ne lui auraient pas permis de se lancer pleinement vers ce nouvel objectif sans remettre partiellement en cause les autres missions qui lui incombent. Et il n'était bien entendu pas question que le développement dece plan sefasse au détriment des activités et pratiques déjà en place ;

- d'autre part, et surtout, parcequel'engagement dans ce projet, au départ porté par un seul médiateur scientifique du CCSTI, a permis d'insuffler une nouvelle dynamique au sein de l'équipe du CCSTI. Des réunions de réflexion impliquant les médiateurs scientifiques et les médiateurs multimédia (Cyber-base) se sont mises en place en vue d'élargir nos offres d'activités de CST pour ce nouveau public

Cette volonté en interne nous a conduit à rencontrer à nouveau le $\mathrm{CIAS}$ afin de croiser les intentions et les besoins de chaque service, et ainsi tenter de conjuguernos projets respectifs dans la meilleure synergie possible.

II nous paraît indispensable d'ouvrir ici une parenthèse intéressante: en effet, alors que nous pensions, àl'occasion decetterencontre,évoquerlesévolutions envisageables de nos médiations en faveur des résidents uniquement, les personnes du CIAS nous ont fait part de la possibilité d'élargir notre champ d'intervention, via le projet de mise en œuvre d'une «plateforme de répit » sur le territoire de l'agglomération d'Annecy.
Développé, porté et animé par le CIAS, ce dispositif, qui devrait être opérationnel dès la rentrée 2013, a pour objectif d'aider et de soutenir les aidants (le termed'aidantrecouvretoutepersonne, professionnelle ou non, qui apporte de l'aide à une personne dépendante) en leur offrant une palette diversifiée de solutions de répit (soutien individualisé, réunions d'information, rencontresconviviales,organisationd'évènements et/ou sorties culturels...). Bien entendu, il n'est pas question que le CCSTI entre dans le processus de développement et de gestion decedispositif, missions qui sont dévolues au CIAS de l'agglomération. En revanche, puisque les activités culturelles font partie de l'éventail d'actions de la plateforme en faveur des aidants, notre structure se pose, à l'instar de chaque institution culturelle du territoire, en partenaire-ressource naturel de ce dispositif. D'un commun accord entre nos deux structures, il a été convenu d'attendre que la plateforme de répit soit parfaitement opérationnelle avant de définir les modalités et formes de ce partenariat particulier.

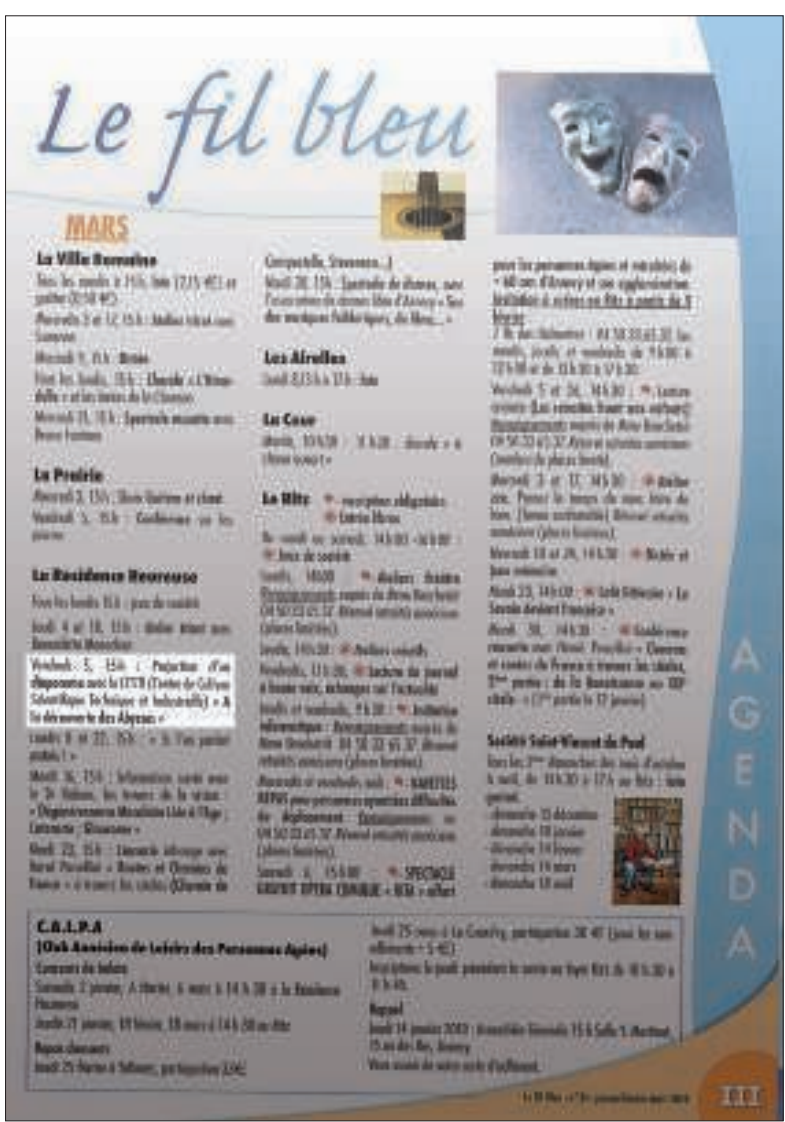

Les activités proposées par La Turbine figurent dans l'agenda du magazine des maisons de retraites de l'agglomération d'Annecy Le fil bleu. o CCSTI La Turbine 
En ce qui concerne les perspectives d'évolution de nos actions de culture scientifique en faveur des résidents, cette réunion a permis à chaque partie d'exprimer librement ses attentes et ses souhaits, d'évoquer les champs d'actions possibles mais également les champs de contraintes inhérents au fonctionnement du CCSTI et des établissements pour personnes âgées, et ainsi d'aboutir à des propositions affinées, et entérinées par tous.

Même si les contenus et outils définitifs restent à créer, il a d'ores et déjà été convenu de part et d'autre qu'ils s'articuleront autour de deux grands axes: les cinq sens d'une part, et l'utilisation d'outils multimédia d'autre part.

Des ateliers d'éveil sensoriel permettronten effetd'appréhender, de manière ludique, des domaines tels que la cognition, la mémoire et l'émotion, tout en s'inscrivant dans une démarche de maintien de lien social et de coopération entre les différents participants.

Sans dévoiler l'intégralité de ceux-ci, nous pouvons toutefois préciser que certains ateliers porteront sur la mise en valeur et l'utilisation d'un seul sens (description d'objets et/ou de matières par le toucher uniquement) alors que d'autres joueront sur le croisement de plusieurs sens (dégustations afin de mettre en évidence les interactions odorat/goût et vue/goût par exemple).

Les outils multimédia, et particulièrement les outils nomadestelsquelestablettestactiles, apparaissent quant à eux comme des supports intéressants pour renouveler ou enrichir les pratiques de médiation. En effet, concernant le travail préparatoire des médiateurs scientifiques et multimédia du CCSTI, la création et/ou la transposition de jeux «classiques » (mots croisés, puzzles, quiz...), ainsi que leur mise en place techniquesurtablettes, apparaissent sensiblement moinschronophagesque pourune réalisation surdes supports traditionnels (fiches imprimées et plastifiées, plateaux...).

Parallèlement, et de façonévidente, l'emploi de cetype de jeux sous forme numérique permet d'éliminer le caractère définitif de leurs versions traditionnelles: ainsi, une fois créés, les jeux numériques pourront être réutilisés, réinitialisés, enrichis et modifiés à volonté et selon les besoins des médiateurs.

Enfin, en plus de cette grande faculté d'adaptation aux impératifs et particularités de chaque animation, les dimensions destablettestactilesautorisentunelogistiqueaiséeetàgéométrievariable,quenepermettent en aucun cas l'emploi de supports traditionnels ou d'ordinateurs portables. Leur emploi dans des activitésà destination depersonnesâgées dépendantes offre ainsi des perspectives très attractives.
Dans ce domaine précis de l'utilisation d'outils multimédia nomades, le CCSTI s'inscrit une fois encore dans l'expérimentation de deux grandes pistes: - desséances dedécouvertebasiqueetpratiquedel'outil tablette en vue d'une utilisation individuelle et personnelle(personnessouhaitantcommuniquerparcourriels avec leur famille et ne pouvant pas se déplacer seules jusqu'à la salle informatique de leur résidence) ; - l'utilisation de tablettes tactiles pour des jeux de types quiz, Memory, Vrai ou Faux... en séances collectives (travail de la cognition et des facultés mémorielles),enappuiouindépendammentd'uneprojection de documentaire.

Précisons à ce point que ces deux grands axes de réflexion ont été soumis aux animatrices de chaque établissement, qui les ont ensuite validés. Les contenus et formats sont maintenant en cours d'élaboration au sein du CCSTI et font l'objet d'une étroite collaboration entremédiateurs scientifiques et médiateurs multimédia, pour une mise en œuvre dès la rentrée 2013.

L'équipe du CCSTI La Turbine reste convaincue que le «tout technologique » ne peut se substituer à la médiation humaine et que l'intégration des TIC ne doit pas se faire par obligation ou par effet de mode. Elle n'enestpasmoins persuadéequel'usagedumultimédia avec des personnes âgées, loin d'être une utopie, estunenécessité, neserait-cequ'auregard desperspectives d'enrichissement et de diversification des pratiques de médiation qu'offrent de tels outils.

\section{En conclusion}

La mise en place et le développement de cette expérience a été très probablement facilitée par le rapprochement qui peut s'instaurer naturellement entre 2 services, la culture et le CIAS, d'une même collectivité territoriale. II n'en reste pas moins que sa réussite est due uniquement à l'engagement et la complicité des personnels des 2 services qui transcendentle «prêtà-porter » institutionnel en « haute culture » adaptée aux contraintes d'un public âgé dépendant. 